\title{
RADIAÇÃO GAMA E ATMOSFERA MODIFICADA PASSIVA NA QUALIDADE DE GOIABAS 'PEDRO SATO'
}

\author{
ANDRÉ JOSÉ DE CAMPOS²; ÉRIKA FUJITA³, LEANDRO CAMARGO NEVES ${ }^{4}$, \\ ROGÉRIO LOPES VIEITES ${ }^{5}$; EDVAN ALVES CHAGAS
}

RESUMO - O objetivo deste trabalho foi avaliar o efeito da irradiação gama associada à atmosfera modificada passiva na qualidade pós-colheita de goiabas 'Pedro Sato', verificando suas características físico-químicas. Foram utilizadas goiabas provenientes da região de Vista Alegre do Alto-SP,Brasil. Após a colheita, as goiabas foram imediatamente transportadas ao Laboratório de Frutas e Hortaliças, pertencente ao Departamento de Gestão e Tecnologia Agroindustrial, da Faculdade de Ciências Agronômicas - UNESP - Câmpus de BotucatuSP, onde foram armazenadas a $10^{\circ} \mathrm{C}$ e 90-95\%UR, em câmara frigorífica, por 28 dias. Os tratamentos foram: controle 1 (sem embalagem e sem irradiação); controle 2 (embalagem de poliestireno (PS) + polietileno de baixa densidade (PEBD) e sem irradiação); tratamento 1 (PS+PEBD e 0,2kGy); tratamento 2 (PS+PEBD e 0,6kGy), e tratamento 3 (PS+PEBD e 1,0kGy). As análises realizadas foram: firmeza, sólidos solúveis (SS), acidez titulável (AT) e índice de maturação. Foi utilizado o delineamento inteiramente casualizado, com esquema fatorial 5 x 5 (tratamento $\mathrm{x}$ tempo). Concluiu-se que as altas doses de irradiação promoveram efeito negativo nas características físico-químicas da goiaba 'Pedro Sato', e que apenas a menor dose utilizada $(0,2 \mathrm{kGy})$ associada à atmosfera modificada conservou frutos com maior qualidade e aceitabilidade, indicado por maiores índice de maturação e teor de sólidos solúveis obtidos.

Termo para indexação: Psidium guajava; atmosfera modificada; armazenamento refrigerado; irradiação, pós-colheita.

\section{GAMMA RADIATION AND PASSIVE MODIFIED ATMOSPHERE ON THE QUALITY OF GUAVAS 'PEDRO SATO'}

\begin{abstract}
The aim of this study was to evaluate the effect of gamma irradiation associated with modified atmosphere, on postharvest quality of guavas 'Pedro Sato', checking their physicochemical characteristics. Guavas from the region of Vista Alegre do Alto/SP/Brazil, were used. After harvest, the fruits were immediately transported to the Fruit and Vegetables Laboratory of the Agroindustrial Management and Technology Department, Agronomic Sciences College - UNESP - Botucatu / SP, where they were kept at $10^{\circ} \mathrm{C}$ and $90-95 \%$ RH in cold storage, for 28 days. The treatments were: control 1 (without package or irradiation), control 2 (polystyrene package-PS) + low density polyethylene package-LDPE and without irradiation) treatment 1 (PS + LDPE and $0.2 \mathrm{kGy}$ ), treatment 2 (PS + LDPE and $0.6 \mathrm{kGy}$ ) and treatment 3 (PS + LDPE and $1.0 \mathrm{kGy}$ ). The analyses were: firmness, soluble solids (SS), titratable acidity (TA) and maturity index. It was used the randomized design with a factorial design $5 \times 5$ (treatment $\mathrm{x}$ time). In the end of this work it was concluded that high doses of irradiation promoted a negative effect on physicochemical characteristics of guava 'Pedro Sato', verifying that only the lowest dose associated with modified atmosphere provided fruits with higher quality and acceptability, due to the higher maturity index and soluble solids obtained.

Index terms: Psidium guajava L., doses, gases, conservation.
\end{abstract}

\footnotetext{
${ }^{1}$ Trabalho Sinfruit 015 - Simpósio Internacional de Fruticultura - Avanços na Fruticultura (17 a 21 Outubro)

${ }^{2}$ Pós-doutorando PNPD/CAPES - UFRR/CCA, BR 174, 69301-970, Boa Vista-RR. E-mail: andre_jc@hotmail.com

${ }^{3}$ Bolsista EXP-A/CNPq - UFRR/CCA, BR 174, 69301-970, Boa Vista-RR. E-mail: rapelbtu@gmail.com

${ }^{4}$ Docente pela UFRR/CCA, BR 174, 69301-970, Boa Vista-RR.

${ }^{5}$ DocentepelaUNESP/FCA,Dpto.GestãoeTecnologiaAgroindustrial,C.Postal237,18160-307,Botucatu-SP.E-mail:svieites@fca.unesp.br

${ }^{6}$ Pesquisador Embrapa Roraima, BR 174, Km 08, 69301-970, Boa Vista-RR. E-mail: echagas@cpafrr.embrapa.br
} 


\section{INTRODUÇÃO}

A goiaba (Psidium guajava L.) é um dos frutos de maior importância nas regiões subtropicais e tropicais, não só devido ao seu elevado valor nutritivo, mas também pela excelente aceitação do consumo in natura, sua grande aplicação industrial, e capacidade de se desenvolver em condições adversas de clima (NEVES, 2009).

O Brasil é um dos maiores produtores mundiais de goiaba, porém a expansão do seu mercado consumidor está condicionado à qualidade dos frutos e à sua vida útil pós-colheita. Altamente perecível, devido ao seu intenso metabolismo durante o amadurecimento, a goiaba tem vida útil de 3 a 5 dias sob temperatura ambiente (GORGATTI NETO et al., 1996). Os principais aspectos de sua deterioração envolvem o rápido amolecimento dos frutos, a perda de coloração verde e do brilho da casca, o murchamento e a incidência de podridões. Tem ainda, como desvantagem a sazonalidade dificulta a oferta durante o ano inteiro, sendo necessário o emprego de processos de conservação que proporcionem tal durabilidade (REIS, 2007).

O armazenamento e a comercialização de produtos vegetais sob refrigeração constituem uma das possibilidades para manter a qualidade do fruto. $\mathrm{O}$ abaixamento da temperatura resulta em redução da atividade respiratória, da transpiração e produção de etileno pelos frutos, com consequente aumento do período de conservação (HARDENBURG et al., 1986).

Diversos tratamentos pós-colheita têm sido testados em goiabas, embora muitos sejam eficientes em retardar a maturação e em conservar a qualidade dos frutos, alguns interferem em suas características sensoriais. Outros estendem a vida útil de forma economicamente inexpressiva, ou deixam resíduos químicos (BASSETO, 2002).

Aradiação gama, associada aosprocedimentos pós-colheita, tem-se mostrado bastante eficiente em prolongar a vida comercial de frutos frescos. Retardam os processos de amadurecimento e senescência, reduzindo o apodrecimento sem provocar alterações significativas em seu aspecto, sabor e qualidade nutritiva (DOMARCO et al., 1996).

Nesse contexto, o objetivo deste trabalho foi avaliar o efeito da irradiação gama, associada à atmosfera modificada passiva, na qualidade póscolheita de goiabas 'Pedro Sato'.

\section{MATERIAL E MÉTODOS}

Foram utilizadas goiabas 'Pedro Sato', provenientes da região de Vista Alegre do AltoSão Paulo,Brasil. Após a colheita, elas foram imediatamente transportadas ao Laboratório de Frutas e Hortaliças, pertencente ao Departamento de Gestão e Tecnologia Agroindustrial, da Faculdade de Ciências Agronômicas - UNESP - Câmpus de Botucatu-SP, onde foram selecionadas quanto ao tamanho e defeitos, visando a uniformizar o lote.

\section{Experimento}

Os tratamentos a que as goiabas 'Pedro Sato'foram submetidas, visando a quantificar o efeito combinado da radiação gama e atmosfera modificada passiva, foram: controle 1 , sem embalagem e sem irradiação; controle 2, embalagem de poliestireno (PS), coberta com filme de polietileno de baixa densidade (PEBD); tratamento 1, PS com PEBD e radiação $0,2 \mathrm{kGy}$; tratamento 2, PS+PEBD e 0,6kGy; e tratamento 3, PS+PEBD e 1,0kGy. A radiação gama foi aplicada após o acondicionamento dos frutos nas embalagens e no mesmo dia da instalação do experimento. As goiabas, após serem submetidas ao tratamento, foram armazenadas em câmara frigoríficas, a $10 \pm 1^{\circ} \mathrm{C}$ e $90-95 \%$ de UR, por 28 dias.

\section{Análises}

Foram realizadas as análises físicoquímicas: firmeza, sólidos solúveis (SS), acidez titulável (AT) e índice de maturação.O delineamento utilizado foi o inteiramente no experimento, foram utilizadas 45 goiabas por tratamento, com 3 goiabas por repetição e 3 repetições em cada dia de análise, por tratamento. As avaliações foram realizadas a cada 7 dias, num período de 28 dias $(0 ; 7 ; 14 ; 21$ e 28 dias). As médias foram comparadas pelo teste de Tukey $(\mathrm{p}<0,05)$.

Firmeza: foi determinada usando-se texturômetro (STEVENS - LFRA texture analyser), com distância de penetração de $15 \mathrm{~mm}$, velocidade de 2,0 mm/seg., e ponteiro TA 9/1000. Procedeu-se à leitura em lados opostos da seção equatorial dos frutos, sendo que o valor obtido para a firmeza em $\mathrm{cN}$ (centinewton) foi definido como a máxima força requerida para que o ponteiro penetrasse na polpa do fruto.

Sólidos solúveis (SS): esse teor foi qualificado através de leitura refratométrica direta, em graus Brix, usando-se refratômetro tipo Palette (ATAGO PR-32), conforme o recomendado pelo IAL (2008).

Acidez titulável (AT): o conteúdo de acidez titulável, expresso em gramas de ácido cítrico por 100 gramas de polpa, foi determinado através da titulação 
de massa conhecida de polpa homogeneizada e diluída com água destilada ou bidestilada, com solução padronizada de hidróxido de sódio a $0,1 \mathrm{M}$, tendo como indicador a fenolftaleína (IAL, 2008).

Índice de maturação: foi determinado pela relação entre o teor de sólidos solúveis e o de acidez titulável (IAL, 2008).

\section{Delineamento estatístico}

O delineamento utilizado foi o inteiramente casualizado, com esquema fatorial 5 x 5 (tratamento $\mathrm{x}$ tempo).

\section{RESULTADOS E DISCUSSÃO}

Em relação ao parâmetro firmeza de polpa das goiabas 'Pedro Sato' (Tabela 1), observou-se interação significativa entre os fatores doses $\mathrm{x}$ tempo. Para as médias das doses de irradiação dentro dos dias de análise $(0 ; 7 ; 14 ; 21$ e 28 dias), observouse que até o sétimo dia foi observado diferença significativa entre as médias, fato não observado até o final do experimento. Oliveira et al. (2006) não verificaram diferença estatística até o sétimo dia de armazenamento, ocorrendo essa significância somente após o $14^{\circ}$ dia. No dia inicial, o tratamento $0,6 \mathrm{kGy}+$ $\mathrm{AM}(\mathrm{PS}+\mathrm{PEDB})$ mostrou as maiores médias, diferindo dos tratamentos 0,2 kGy + AM e 1,0 kGy + AM, mas não diferindo dos demais. No $7^{\circ}$ dia de análise, o tratamento-controle 2 proporcionou as maiores médias de firmeza de polpa, diferindo estatisticamente dos demais, exceção apenas para o 0,6 kGy + AM. Nos demais dias de análise, mesmo sem a ocorrência da diferença significativa entre os tratamentos, verificaram-se as maiores médias para o controle + AM, o que é semelhante ao encontrado por Pimentel (2007), onde observou que todas as doses de irradiação gama testadas promoveram redução na firmeza das goiabas 'Pedro Sato' durante o armazenamento. Esses resultados são discordantes dos encontrados por Oliveira et al. (2006), que trabalhando com goiaba 'Kumagai', observaram que os frutos tratados com a dose $0,6 \mathrm{kGy}$ mostraram-se significativamente mais firmes que os demais. Essa perda de firmeza dos frutos irradiados pode ser devida à alta sensibilidade das goiabas à aplicação da irradiação gama, bem como à atividade das enzimas hidrolíticas, como a poligalacturonase e pectinametilesterase (JAIN et al., 2001), que promovem intensa solubilização das pectinas constituintes da parede celular (TUCKER, 1993).

Analisando as médias dos dias de análise dentro das doses de irradiação, observou-se a constante redução da firmeza até o final do período experimental em todos os tratamentos, onde o dia inicial proporcionou as maiores médias desse parâmetro diferindo estatisticamente dos demais, o que também foi observado por Cavalini (2004) trabalhando com goiabas 'Kumagai' e 'Paluma'.

$\mathrm{Na}$ Tabela 2, referente à acidez titulável, observou-se a interação dupla significativa entre os fatores doses $\mathrm{x}$ tempo. Com relação às médias dentro dos dias de análise, verificou-se a não ocorrência de diferença estatística significativa no período experimental, exceção apenas para o $28^{\circ}$ dia. No final do período de armazenamento, o tratamento-controle + AM proporcionou a maior média desse parâmetro em relação ao controle, não diferindo dos demais tratamentos aplicados. Esse resultado corrobora o de com Pimentel (2007), que trabalhou com goiabas 'Pedro Sato' e verificou que a irradiação gama não causou alteração na acidez .A diferença observada no experimento foi devida aos frutos-controle terem sido descartados no final do experimento, por estarem em estágio avançado de senescência. Esse resultado é diferente do relatado por Oliveira et al. (2006), ao verificarem que as goiabas 'Kumagai' tratadas com a dose $0,6 \mathrm{kGy}$ e o controle aumentaram dos valores de acidez titulável no $14^{\circ}$ dia e no final do período de armazenamento, respectivamente.

Para as médias dos dias de análise nas doses de irradiação gama, observou-se a ocorrência de diferença estatística somente nos frutos-controle, em que o último dia de análise propiciou as menores médias. Essa menor média observada no $28^{\circ}$ dia é causada pelo descarte do tratamento-controle, frutos que estavam em estágio avançado de senescência. Outro fato importante ocorrido, nas condições deste experimento, foi a oscilação dos valores médios de acidez titulável, que foi atribuído à grande sensibilidade das goiabas à irradiação gama e atmosfera modificada. Estes resultados discordam de Carvalho (1999) e Gianonni (2000), que observaram aumento na acidez titulável em goiabas armazenadas sob refrigeração. Os valores médios obtidos encontraram-se entre 0,82 e $1,00 \mathrm{~g}$ ácido cítrico $100 \mathrm{~g}^{-1}$, sendo superiores aos encontrados por Silva et al. (2009), que relataram valores entre 0,50 e $0,53 \mathrm{~g}$ ácido cítrico $100 \mathrm{~g}^{-1}$ para a cultivar 'Paluma'.

Em relação aos teores de sólidos solúveis (Tabela 3), observou-se interação significativa entre os fatores doses de irradiação x dias de análise. Para as médias das doses de irradiação dentro dos dias de análise, observou-se a ocorrência de diferença significativa entre as médias desse parâmetro nos dias $0 ; 7 ; 14$ e 28 dias de avaliação, com exceção do $14^{\circ}$ e $21^{\circ}$ dias. No dia inicial, o tratamento-controle mostrou as maiores médias, diferindo somente do tratamento 0,6 
$\mathrm{kGy}+\mathrm{AM}$, mas não diferindo dos demais. No $7^{\circ}$ dia de análise, o tratamento-controle + AM proporcionou as maiores médias, diferindo apenas do 1,0 kGy + AM. Com relação ao $28^{\circ}$ dia, ocorreu inversão dos valores médios, onde o tratamento $1,0 \mathrm{kGy}+\mathrm{AM}$ propiciou as maiores médias de sólidos solúveis, diferindo somente do controle.Isto é diferente do relatado por Oliveira et al. (2006), que observaram que os frutos tratados com as menores doses de radiação propiciaram os menores valores desse parâmetro no início do período experimental. Essa inversão nos valores médios é devido à perda de água por transpiração, em que os tratamentos que sofreram a aplicação da irradiação + atmosfera modificada conseguiram manter a qualidade do fruto por mais tempo, em comparação ao controle, evitando a perda de água das goiabas.

Analisando as médias dos dias de análise dentro das doses de irradiação, observou-se a ocorrência de diferença estatística somente nos tratamentos-controle e controle $+\mathrm{AM}$, não sendo verificada tal ocorrência nos demais tratamentos. Para o tratamento-controle, $\mathrm{o} 14^{\circ}$ dia de analise proporcionou as maiores médias em comparação ao $21^{\circ}$ e $28^{\circ}$ dia, mas não diferindo do dia inicial e do sétimo. No caso do tratamento-controle $+\mathrm{AM}$, o $7^{\circ}$ dia diferiu estatisticamente do $21^{\circ}$, mostrando as maiores médias. Nesse contexto, $\mathrm{o} 14^{\circ}$ dia propiciou os maiores valores de sólidos solúveis, sendo que após esse dia ocorreu redução nos valores até o final do experimento. Essa redução ocorre, pois os frutos utilizam os açúcares no processo de respiração, promovendo a diminuição no teor de sólidos solúveis, o que também foi observado t por Mattiuz e Durigan (2003) e Pimentel (2007. Os valores médios de sólidos solúveis encontrados neste experimento situam-se entre 8,60 a $10,37^{\circ} \mathrm{Brix}$, que são superiores aos encontrados por Silva et al. (2009), que observaram valores entre 8,18 a $8,89^{\circ}$ Brix para a cultivar Paluma e Azzolini et al. (2004), que relataram valores de $7,60^{\circ}$ Brix para a cultivar Pedro Sato.

$\mathrm{Na}$ Tabela 4, referente ao Índice de Maturação dos frutos, observou-se a interação dupla significativa entre os fatores doses de irradiação gama $\mathrm{x}$ dias de análise. Com relação às médias das doses de irradiação gama dentro dos dias de análise, verificou-se a não ocorrência de diferença estatística durante todo o período experimental. A menor média relatada no $28^{\circ}$ dia é causada pelo descarte do tratamento-controle, pois os frutos que estavam em estágio avançado de senescência. O tratamento 0,2 $\mathrm{kGy}+\mathrm{AM}$ proporcionou o maior índice de maturação nos frutos de goiaba.

Para as médias dos dias de análise nas doses de irradiação gama, não se observou a ocorrência de diferença estatística. Nas condições em que foi instalado este experimento, foi possível constatar a oscilação dos valores médios para esse parâmetro, o que é diferente do observado por Frateschi (1999) e Oliveira et al. (2006), que reltaram a diminuição do índice de maturação das goiabas 'Kumagai' no decorrer do período de armazenamento.

Os valores médios estiveram entre 9,15 e 12,05 , sendo abaixo do valor 16,12 encontrado por Azollini et al. (2004) para goiabas 'Pedro Sato' e bem abaixo dos resultados 24,80 e 30,80 encontrados por Manica et al. (1998) para cultivares 'Pirassunungavermelha' e 'IAC4', respectivamente.

TABELA 1 - Firmeza de Polpa de goiabas 'Pedro Sato' irradiadas com Cobalto 60 e armazenadas a $10^{\circ} \mathrm{C}$ e 90-95\% UR. Boa Vista, UFRR, 2010.

\begin{tabular}{ccccccc}
\hline & \multicolumn{7}{c}{ Firmeza de Polpa (cN) } \\
\cline { 2 - 7 } Tratamentos & controle & controle + & $\mathbf{0 , 2} \mathbf{~ k G y}+$ & $\mathbf{0 , 6} \mathbf{~ k G y}+$ & $\mathbf{1 , 0} \mathbf{~ k G y}+$ & Média \\
& & $\mathbf{A M}$ & $\mathbf{A M}$ & $\mathbf{A M}$ & $\mathbf{A M}$ & \\
\hline $\mathbf{0}$ dia & $724,56 \mathrm{Aab}$ & $763,42 \mathrm{Aa}$ & $640,50 \mathrm{Ab}$ & $778,17 \mathrm{Aa}$ & $472,83 \mathrm{Ac}$ & $675,89 \mathrm{a}$ \\
$\mathbf{7}$ dias & $163,44 \mathrm{Bb}$ & $343,22 \mathrm{Ba}$ & $200,72 \mathrm{Bb}$ & $252,05 \mathrm{Bab}$ & $208,22 \mathrm{Bb}$ & $233,53 \mathrm{~b}$ \\
$\mathbf{1 4}$ dias & $121,28 \mathrm{BCa}$ & $154,17 \mathrm{Ca}$ & $78,34 \mathrm{Ca}$ & $70,06 \mathrm{Ca}$ & $109,11 \mathrm{Ca}$ & $106,59 \mathrm{c}$ \\
$\mathbf{2 1}$ dias & $65,94 \mathrm{CDa}$ & $66,33 \mathrm{Ca}$ & $36,72 \mathrm{Ca}$ & $43,83 \mathrm{Ca}$ & $49,39 \mathrm{Ca}$ & $52,44 \mathrm{~d}$ \\
$\mathbf{2 8}$ dias & $0,00 \mathrm{Da}$ & $65,61 \mathrm{Ca}$ & $42,61 \mathrm{Ca}$ & $23,78 \mathrm{Ca}$ & $29,50 \mathrm{Ca}$ & $32,30 \mathrm{~d}$ \\
\hline Média & $215,04 \mathrm{BC}$ & $278,55 \mathrm{~A}$ & $199,78 \mathrm{BC}$ & $233,58 \mathrm{~B}$ & $173,81 \mathrm{C}$ & \\
\hline
\end{tabular}

Letras minúsculas comparam médias de doses de irradiação gama para cada dia de análise (linha);

Letras maiúsculas comparam médias nos dias de análise para cada dose de irradiação gama (coluna);

Médias seguidas pela mesma letra não diferem significativamente entre si, a 5\% de probabilidade, pelo teste de Tukey. 
TABELA 2 - Acidez Titulável de goiabas 'Pedro Sato' irradiadas com Cobalto 60 e armazenados à 10C e 90-95\% UR. Boa Vista, UFRR, 2010.

\begin{tabular}{ccccccc}
\hline & \multicolumn{5}{c}{ Acidez Titulável (g ácido cítrico 100g-1 p.f.) } \\
\cline { 2 - 7 } Tratamentos & controle & $\begin{array}{c}\text { controle }+ \\
\mathbf{A M}\end{array}$ & $\begin{array}{c}\mathbf{0 , 2} \mathbf{~ k G y}+ \\
\mathbf{A M}\end{array}$ & $\begin{array}{c}\mathbf{0 , 6} \mathbf{~ k G y}+ \\
\mathbf{A M}\end{array}$ & $\begin{array}{c}\mathbf{1 , 0} \mathbf{~ k G y}+ \\
\mathbf{A M}\end{array}$ & Média \\
& & $0,93 \mathrm{Aa}$ & $0,84 \mathrm{Aa}$ & $0,91 \mathrm{Aa}$ & $0,86 \mathrm{Aa}$ & $0,91 \mathrm{a}$ \\
$\mathbf{0 ~ d i a}$ & $1,00 \mathrm{Aa}$ & $0,99 \mathrm{Aa}$ & $0,92 \mathrm{Aa}$ & $0,96 \mathrm{Aa}$ & $0,88 \mathrm{Aa}$ & $0,92 \mathrm{a}$ \\
$\mathbf{7}$ dias & $0,82 \mathrm{Aa}$ & $0,88 \mathrm{Aa}$ & $0,94 \mathrm{Aa}$ & $0,92 \mathrm{Aa}$ & $0,89 \mathrm{Aa}$ & $0,91 \mathrm{a}$ \\
$\mathbf{1 4}$ dias & $0,94 \mathrm{Aa}$ & $0,88 \mathrm{Aa}$ & $0,97 \mathrm{Aa}$ & $0,97 \mathrm{Aa}$ & $0,91 \mathrm{Aa}$ & $0,93 \mathrm{a}$ \\
$\mathbf{2 1}$ dias & $0,91 \mathrm{Aa}$ & $0,80,0,86 \mathrm{Aa}$ & $0,72 \mathrm{~b}$ \\
$\mathbf{2 8}$ dias & $0,00 \mathrm{Bb}$ & $1,00 \mathrm{Aa}$ & $0,82 \mathrm{Aa}$ & $0,91 \mathrm{Aa}$ & 0,86 \\
\hline Média & $0,74 \mathrm{~B}$ & $0,94 \mathrm{~A}$ & $0,90 \mathrm{~A}$ & $0,93 \mathrm{~A}$ & $0,88 \mathrm{~A}$ & \\
\hline
\end{tabular}

Letras minúsculas comparam médias de doses de irradiação gama para cada dia de análise (linha);

Letras maiúsculas comparam médias nos dias de análise para cada dose de irradiação gama (coluna);

Médias seguidas pela mesma letra não diferem significativamente entre si, a $5 \%$ de probabilidade, pelo teste de Tukey.

TABELA 3 - Teores de Sólidos Solúveis ( ${ }^{\circ}$ Brix) em goiabas 'Pedro Sato' irradiadas com Cobalto 60 e armazenadas a $10^{\circ} \mathrm{C}$ e $90-95 \%$. Boa Vista, UFRR, 2010.

\begin{tabular}{ccccccc}
\hline \multirow{2}{*}{ Tratamentos } & \multicolumn{5}{c}{ Sólidos Solúveis ( $\left.{ }^{\mathbf{} B r i x}\right)$} \\
\cline { 2 - 7 } & \multirow{2}{*}{ controle } & $\begin{array}{c}\text { controle }+ \\
\mathbf{A M}\end{array}$ & $\mathbf{0 , 2} \mathbf{~ k G y}+$ & $\mathbf{0 , 6} \mathbf{k G y}+$ & $\mathbf{1 , 0} \mathbf{~ k G y}+$ & \multirow{2}{*}{ Média } \\
& $10,10 \mathrm{ABa}$ & $9,50 \mathrm{ABab}$ & $10,03 \mathrm{Aa}$ & $8,83 \mathrm{Ab}$ & $9,53 \mathrm{Aab}$ & $9,60 \mathrm{ab}$ \\
$\mathbf{0}$ dia & $9,87 \mathrm{ABa}$ & $10,10 \mathrm{Aa}$ & $9,93 \mathrm{Aa}$ & $9,87 \mathrm{Aa}$ & $8,60 \mathrm{Ab}$ & $9,67 \mathrm{ab}$ \\
$\mathbf{7}$ dias & $10,37 \mathrm{Aa}$ & $9,87 \mathrm{ABa}$ & $10,17 \mathrm{Aa}$ & $9,67 \mathrm{Aa}$ & $9,67 \mathrm{Aa}$ & $9,95 \mathrm{a}$ \\
$\mathbf{1 4}$ dias & $9,23 \mathrm{Ba}$ & $8,90 \mathrm{Ba}$ & $9,50 \mathrm{Aa}$ & $9,27 \mathrm{Aa}$ & $9,57 \mathrm{Aa}$ & $9,29 \mathrm{~b}$ \\
$\mathbf{2 8}$ dias & $0,00 \mathrm{Cb}$ & $9,13 \mathrm{ABa}$ & $9,20 \mathrm{Aa}$ & $9,30 \mathrm{Aa}$ & $9,37 \mathrm{Aa}$ & $7,40 \mathrm{c}$ \\
\hline Média & $7,91 \mathrm{~B}$ & $9,50 \mathrm{~A}$ & $9,77 \mathrm{~A}$ & $9,39 \mathrm{~A}$ & $9,35 \mathrm{~A}$ & \\
\hline
\end{tabular}

Letras minúsculas comparam médias de doses de irradiação gama para cada dia de análise (linha);

Letras maiúsculas comparam médias nos dias de análise para cada dose de irradiação gama (coluna);

Médias seguidas pela mesma letra não diferem significativamente entre si, a 5\% de probabilidade, pelo teste de Tukey.

TABELA 4 - Índice de Maturação em goiabas 'Pedro Sato' irradiadas com Cobalto 60 e armazenadas a $10 \pm 1^{\circ} \mathrm{C}$ e $90-95 \%$ UR. Boa Vista, UFRR, 2010

\begin{tabular}{ccccccc}
\hline \multirow{2}{*}{ Tratamentos } & \multicolumn{7}{c}{ Índice de Maturação } \\
\cline { 2 - 7 } & controle & $\begin{array}{c}\text { controle }+ \\
\text { AM }\end{array}$ & $\begin{array}{c}\mathbf{0 , 2} \mathbf{~ k G y}+ \\
\mathbf{A M}\end{array}$ & $\mathbf{0 , 6} \mathbf{k G y}+$ & $\mathbf{1 , 0} \mathbf{~ k G y}+$ & Média \\
\hline $\mathbf{0}$ dia & $10,30 \mathrm{Aa}$ & $10,19 \mathrm{Aa}$ & $12,00 \mathrm{Aa}$ & $9,92 \mathrm{Aa}$ & $11,31 \mathrm{Aa}$ & $10,75 \mathrm{a}$ \\
$\mathbf{7}$ dias & $12,05 \mathrm{Aa}$ & $10,24 \mathrm{Aa}$ & $10,87 \mathrm{Aa}$ & $10,25 \mathrm{Aa}$ & $9,77 \mathrm{Aa}$ & $10,64 \mathrm{a}$ \\
$\mathbf{1 4}$ dias & $11,08 \mathrm{Aa}$ & $11,22 \mathrm{Aa}$ & $11,01 \mathrm{Aa}$ & $10,68 \mathrm{Aa}$ & $10,89 \mathrm{Aa}$ & $10,98 \mathrm{a}$ \\
$\mathbf{2 1}$ dias & $10,23 \mathrm{Aa}$ & $10,11 \mathrm{Aa}$ & $9,81 \mathrm{Aa}$ & $9,62 \mathrm{Aa}$ & $10,57 \mathrm{Aa}$ & $10,07 \mathrm{a}$ \\
$\mathbf{2 8}$ dias & $0,00 \mathrm{Bb}$ & $9,15 \mathrm{Aa}$ & $11,47 \mathrm{Aa}$ & $10,22 \mathrm{Aa}$ & $10,99 \mathrm{Aa}$ & $8,37 \mathrm{~b}$ \\
\hline Média & $8,73 \mathrm{~B}$ & $10,18 \mathrm{~A}$ & $11,03 \mathrm{~A}$ & $10,14 \mathrm{~A}$ & $10,71 \mathrm{~A}$ & \\
\hline
\end{tabular}

Letras minúsculas comparam médias de doses de irradiação gama para cada dia de análise (linha);

Letras maiúsculas comparam médias nos dias de análise para cada dose de irradiação gama (coluna);

Médias seguidas pela mesma letra não diferem significativamente entre si, a $5 \%$ de probabilidade, pelo teste de Tukey. 


\section{CONCLUSÃO}

Nas condições em que foi instalado o experimento, conclui-se que altas doses de irradiação ( 0,6 e 1,0 kGy) promoveram efeito negativo nas características físico-químicas da goiaba 'Pedro Sato'. A menor dose $(0,2 \mathrm{kGy})$ associada à atmosfera modificada proporcionou frutos com maiores índice de maturação e teores de sólidos solúveis obtidos.

\section{REFERÊNCIAS}

AZZOLINI, M.; JACOMINO, A.P.; SPOTO, M.H.F. Estádios de maturação e qualidade pós-colheita de goiabas 'Pedro Sato'. Revista Brasileira de Fruticultura, Jaboticabal, v.26, p.29-31, 2004.

BASSETO, E. Conservação de goiabas "Pedro Sato" tratadas com 1-metilciclopropeno: concentrações e tempos de exposição. 2002. 71 f. Dissertação (Mestrado) - Escola Superior de Agricultura “Luiz de Queiroz”, Universidade de São Paulo, Piracicaba, 2002.

CARVALHO, H.A. de. Utilização de atmosfera modificada na conservação pós-colheita da goiaba "Kumagai". Lavras, 1999. 115 f. Tese (Doutorado em Ciências dos Alimentos) - Universidade Federal de Lavras, Lavras, 1999.

CAVALINI, F.C. Índices de maturação, ponto de colheita e padrão respiratório de goiabas 'Kumagai' e 'Paluma'. 2004. 69f. Dissertação (Mestrado) - Escola Superior de Agricultura Luiz de Queiroz, Universidade de São Paulo, Piracicaba, 2004.

DOMARCO, R.E. et al. Inibição do amadurecimento de bananas por radiação gama: aspectos físicos, químicos e sensoriais. Ciência e Tecnologia de Alimentos, Campinas, v.16, n.2, p.137-142, 1996.

FRATESCHI, P.W.B. Radiação gama com ${ }^{60}$ cobalto na conservação pós-colheita de goiaba-branca. 1999. 141f. Dissertação (Mestrado) - Universidade Federal de Goiás, Goiânia, 1999.

GIANNONI, J. A. Efeito da radiação gama e do cálcio na conservação pós-colheita da goiababranca armazenada sob refrigeração. 2000. $181 \mathrm{f}$. Dissertação (Mestrado em Agronomia) - Faculdade de Ciências Agronômicas,Universidade Estadual Paulista, Botucatu, 2000.
GORGATTI NETTO, A.; GARCIA, A. E.; ARDITO, E. F. G. Goiaba para exportação: procedimentos de colheita e pós-colheita. Brasília: Embrapa-SPI, 1996. 35 p. (Publicações Técnicas FRUPEX, 20).

HANDERBURG, R.E.; WATADA, A.E.; WANG, C.Y. The commercial storage of fruits, vegetables, and florist, and nursery stocks. Washington: USDA, 1986. 130 p. (Agriculture Handbook, 66).

INSTITUTO ADOLFO LUTZ. Métodos físicoquímicos para análise de alimentos. São Paulo, 2008. p. 1020.

JAIN, N. et al. Compositional and enzymatic changes in guava (Psidium guajava L.) fruits during ripening. Acta Physiologiae Plantarum, Kraków v.23, p. 357-362, 2001.

MANICA, I.; KIST, H.; MICHELETTO, E.L.; KRAUSE, C.A. Competição entre quatro cultivares e duas seleções de goiabeira. Pesquisa Agropecuária Brasileira, Brasília, v.33, n.8, p.1305-1313, 1998.

MATTIUZ, B.; DURIGAN, J.F. Fisiologia e qualidade pós-colheita de goiabas 'Paluma'e 'Pedro Sato', submetidas à injúria mecânica por impacto. Revista Brasileira de Armazenamento, Viçosa, MG, v.28, n.1, p. 46-50, 2003.

NEVES, L.C. (Org.). Manual Pós-Colheita da fruticultura brasileira. Londrina: EDUEL, 2009. $494 \mathrm{p}$.

OLIVEIRA, A.C.G. et al. Conservação pós-colheita de goiaba-branca 'Kumagai' por irradiação gama: aspectos físicos, químicos e sensoriais. Boletim CEPPA, Curitiba, v. 24, n. 2, p. 375-396, jul./dez. 2006.

PIMENTEL, R.M.A. Qualidade pós-colheita da goiaba-vermelha (Psidium guajava $\mathrm{L}$.) submetida ao tratamento quarentenário por irradiação gama. 2007. 112f. Tese (Doutorado em Ciências, Área de Concentração: Energia Nuclear na Agricultura) - Centro de Energia Nuclear na Agricultura da Universidade de São Paulo, Piracicaba, 2007.

REIS, K.C. dos et al. Avaliação físico-química de goiabas desidratadas osmoticamente em diferentes soluções. Ciência e Agrotecnologia, Lavras, v. 31, n. 3, p. 781-785, 2007. 
SILVA, E.C.; MAGALHÃES, C.H.; GONÇALVES， TUCKER, G.A.; Introduction. In: SEYMOUR,G.B.; R.A. Obtenção e avaliação de parâmetros físico- TAYLOR,J.E.; TUCKER, G.A. (Ed.). Biochemistry químicos da polpa de goiaba (Psidium guajava L.), of fruit ripening. London: Champman \& Hall, 1993. cultivar 'Paluma'. In: SEMANA DE CIÊNCIA E TECNOLOGIA DO IFMG, 2.; JORNADA p. 2-51. CIENTIFICA, 2, 2009, Campus Bambuí. Anais... 